\title{
Weighted Integrals of Holomorphic Functions on the Polydisc II
}

\author{
Stevo Stević
}

Abstract. Let $\mathcal{L}_{\alpha}^{p}\left(U^{n}\right)$ denote the class of all measurable functions defined on the unit polydisc $U^{n}=\left\{z \in \mathbf{C}^{n}|| z_{i} \mid<1, i=1, \ldots, n\right\}$ such that

$$
\|f\|_{\mathcal{L}_{\alpha}\left(U^{n}\right)}^{p}=\int_{U^{n}}|f(z)|^{p} \prod_{j=1}^{n}\left(1-\left|z_{j}\right|^{2}\right)^{\alpha_{j}} d m\left(z_{j}\right)<\infty
$$

where $\alpha_{j}>-1, j=1, \ldots, n$, and $d m\left(z_{j}\right)$ is the normalized area measure on the unit disk $U, H\left(U^{n}\right)$ the class of all holomorphic functions on $U^{n}$, and let $\mathcal{A}_{\alpha}^{p}\left(U^{n}\right)=$ $\mathcal{L}_{\alpha}^{p}\left(U^{n}\right) \cap H\left(U^{n}\right)$ (the weighted Bergman space). In this paper we prove that for $p \in(0, \infty), f \in \mathcal{A}_{\alpha}^{p}\left(U^{n}\right)$ if and only if the functions

$$
\prod_{j \in S}\left(1-\left|z_{j}\right|^{2}\right) \frac{\partial^{|S|} f}{\prod_{j \in S} \partial z_{j}}\left(\chi_{S}(1) z_{1}, \chi_{S}(2) z_{2}, \ldots, \chi_{S}(n) z_{n}\right)
$$

belong to the space $\mathcal{L}_{\alpha}^{p}\left(U^{n}\right)$ for every $S \subseteq\{1,2, \ldots, n\}$, where $\chi_{S}(\cdot)$ is the characteristic function of $S,|S|$ is the cardinal number of $S$, and $\prod_{j \in S} \partial z_{j}=\partial z_{j_{1}} \cdots \partial z_{j_{|S|}}$, where $j_{k} \in S, k=1, \ldots,|S|$. This result extends Theorem 22 of Kehe Zhu in Trans. Amer. Math. Soc. 309 (1988) (1), $253-268$, when $p \in(0,1)$. Also in the case $p \in[1, \infty)$, we present a new proof.

Keywords: Holomorphic function, weighted Bergman space, polydisc

MSC 2000: Primary 32A10, secondary 32A36

\section{Introduction}

Let $z=\left(z_{1}, \ldots, z_{n}\right)$ and $w=\left(w_{1}, \ldots, w_{n}\right)$ be points in complex vector space $\mathbf{C}^{n}$, $U$ the unit disc in the complex plane $\mathbf{C}, U^{n}$ the open unit polydisc in $\mathbf{C}^{n}$ and let $H\left(U^{n}\right)$ be the class of all holomorphic functions $f$ defined on $U^{n}$.

Stevo Stević: Mathematical Institute of Serbian Academy of Science, Knez Mihailova 35/I, 11000 Beograd, Serbia; sstevic@ptt.yu and sstevo@matf.bg.ac.yu

ISSN 0232-2064 / \$2.50 C Heldermann Verlag Berlin 
Let $\gamma=\left(\gamma_{1}, \ldots, \gamma_{n}\right)$ be a multi-index, $\gamma_{k}$ being nonnegative integers, we write

$$
|\gamma|=\gamma_{1}+\cdots+\gamma_{n}, \quad \gamma !=\gamma_{1} ! \cdots \gamma_{n} !, \quad z^{\gamma}=z_{1}^{\gamma_{1}} \cdots z_{n}^{\gamma_{n}} .
$$

For a holomorphic function $f$ we denote

$$
D^{\gamma} f=\frac{\partial^{|\gamma|} f}{\partial z_{1}^{\gamma_{1}} \cdots \partial z_{n}^{\gamma_{n}}} .
$$

Let $\alpha=\left(\alpha_{1}, \ldots, \alpha_{n}\right)$, with $\alpha_{j}>-1$ for $j=1, \ldots, n$, and $p \in(0, \infty)$. The space $\mathcal{L}_{\alpha}^{p}\left(U^{n}\right)=\mathcal{L}_{\alpha}^{p}$ denotes the class of all measurable functions defined on the polydisc $U^{n}$ such that

$$
\|f\|_{\mathcal{L}_{\alpha}}^{p}=\int_{U^{n}}|f(z)|^{p} \prod_{j=1}^{n}\left(1-\left|z_{j}\right|^{2}\right)^{\alpha_{j}} d m\left(z_{j}\right)<\infty
$$

where $d m\left(z_{j}\right)=\frac{1}{\pi} r_{j} d r_{j} d \theta_{j}$ is the normalized area measure on the unit disk $U$. When $\alpha=\overrightarrow{0}$ we denote the space by $\mathcal{L}^{p}\left(U^{n}\right)$. The weighted Bergman space $\mathcal{A}_{\alpha}^{p}\left(U^{n}\right)=\mathcal{A}_{\alpha}^{p}$ is the intersection of $\mathcal{L}_{\alpha}^{p}$ and $H\left(U^{n}\right)$.

Weighted Bergman spaces of analytic functions of one variable have been studied, for example, in $[3-6]$ and $[11,12,16,19]$ while the weighted Bergman spaces of analytic functions on the unit ball $B \subset \mathbf{C}^{n}$ have been studied, for example, in [1], [7-10] and [15, 17, 18] (see, also the references therein).

Throughout the rest of the paper $S$ denotes a subset of $\{1, \ldots, n\}, \chi_{S}(\cdot)$ is the characteristic function of $S,|S|$ denotes the cardinal number of $S$, and $\prod_{j \in S} \partial z_{j}=\partial z_{j_{1}} \cdots \partial z_{j_{|S|}}$, where $j_{k} \in S, k=1, \ldots,|S|$.

In $[1$, p. 33] and [15] the authors proved the following theorem.

Theorem A. Let $p \in(0, \infty), \alpha=\left(\alpha_{1}, \ldots, \alpha_{n}\right)$, with $\alpha_{j}>-1$ for $j=1, \ldots, n$, let $m$ be a fixed positive integer and $\mathbf{k}=\left(k_{1}, \ldots, k_{n}\right) \in\left(\mathbf{Z}_{+}\right)^{n}$. Let $f \in H\left(U^{n}\right)$, then $f \in \mathcal{A}_{\alpha}^{p}$ if and only if

$$
\left[\prod_{j=1}^{n}\left(1-\left|z_{j}\right|^{2}\right)^{k_{j}}\right] \frac{\partial^{|\mathbf{k}|} f}{\partial z_{1}^{k_{1}} \cdots \partial z_{n}^{k_{n}}}(z) \in \mathcal{L}_{\alpha}^{p} \quad \forall \mathbf{k},|\mathbf{k}|=m .
$$

Moreover,

$$
\|f\|_{\mathcal{A}_{\alpha}^{p}} \asymp \sum_{\mid \mathbf{k}=0}^{m-1}\left|\frac{\partial^{|\mathbf{k}|} f}{\partial z_{1}^{k_{1}} \cdots \partial z_{n}^{k_{n}}}(0)\right|+\sum_{|\mathbf{k}|=m}\left\|\left[\prod_{j=1}^{n}\left(1-\left|z_{j}\right|^{2}\right)^{k_{j}}\right] \frac{\partial^{m} f}{\partial z_{1}^{k_{1}} \cdots \partial z_{n}^{k_{n}}}\right\| \|_{\mathcal{L}_{\alpha}^{p}} .
$$

The above statement means that there are finite positive constants $C$ and $C^{\prime}$ independent of $f$ such that the left and right hand sides $L(f)$ and $R(f)$ satisfy

$$
C R(f) \leq L(f) \leq C^{\prime} R(f)
$$


for all holomorphic $f$.

In the proof of Theorem A, when $p \in[1, \infty)$, G. Benke and D. C. Chang used the weighted Bergman projection $\mathbf{B}_{\alpha}: \mathcal{L}_{\alpha}^{2} \rightarrow \mathcal{A}_{\alpha}^{2}$, which can be extended as a bounded operator from $\mathcal{L}_{\alpha}^{p}$ onto $\mathcal{A}_{\alpha}^{p}$. Case $p \in(0,1]$ was considered by quite different method by author of this paper in [15].

Using also a Bergman type projection K. Zhu in [17] proved the following result.

Theorem B. Suppose $1 \leq p<\infty$ and $f \in H\left(U^{2}\right)$, then $f \in \mathcal{A}^{p}\left(U^{2}\right)$ if and only if the functions

$$
\begin{aligned}
T_{1} f\left(z_{1}, 0\right) & =\left(1-\left|z_{1}\right|^{2}\right) \frac{\partial f\left(z_{1}, 0\right)}{\partial z_{1}} \\
T_{2} f\left(0, z_{2}\right) & =\left(1-\left|z_{2}\right|^{2}\right) \frac{\partial f\left(0, z_{2}\right)}{\partial z_{2}} \\
T_{3} f\left(z_{1}, z_{2}\right) & =\left(1-\left|z_{1}\right|^{2}\right)\left(1-\left|z_{2}\right|^{2}\right) \frac{\partial^{2} f\left(z_{1}, z_{2}\right)}{\partial z_{1} \partial z_{2}}
\end{aligned}
$$

are in $\mathcal{L}^{p}\left(U^{2}\right)$. Moreover, $\|\cdot\|_{\mathcal{A}^{p}}$ and

$$
\|f\|_{*}=|f(0,0)|+\left\|T_{1} f\right\|_{\mathcal{L}^{p}}+\left\|T_{2} f\right\|_{\mathcal{L}^{p}}+\left\|T_{3} f\right\|_{\mathcal{L}^{p}}
$$

are equivalent norms on $\mathcal{A}^{p}\left(U^{2}\right)$.

Closely related results on the unit disc and the unit ball in $\mathbf{C}^{n}$ or $\mathbf{R}^{n}$ can be found in $[1-3]$ and $[10-18]$. The purpose of this note is to generalize Theorem B in the case $p \in(0,1)$. We prove the following theorem.

Theorem 1. Let $p \in(0, \infty), \alpha=\left(\alpha_{1}, \ldots, \alpha_{n}\right)$, with $\alpha_{j}>-1$ for $j=1, \ldots, n$, and $f \in H\left(U^{n}\right)$. Then $f \in \mathcal{A}_{\alpha}^{p}\left(U^{n}\right)$ if and only if the functions

$$
T_{S} f=\prod_{j \in S}\left(1-\left|z_{j}\right|^{2}\right) \frac{\partial^{|S|} f}{\prod_{j \in S} \partial z_{j}}\left(\chi_{S}(1) z_{1}, \chi_{S}(2) z_{2}, \ldots, \chi_{S}(n) z_{n}\right)
$$

belong to the space $\mathcal{L}_{\alpha}^{p}\left(U^{n}\right)$ for every $S \subseteq\{1,2, \ldots, n\}$. Moreover, $\|\cdot\|_{\mathcal{A}^{p}}$ and $\|\cdot\|_{*}$ are equivalent norms on $\mathcal{A}^{p}\left(U^{n}\right)$, where

$$
\|f\|_{*}=|f(\overrightarrow{0})|+\sum_{S \subseteq\{1, \ldots, n\}, S \neq \emptyset}\left\|T_{S} f\right\|_{\mathcal{L}_{\alpha}^{p}}
$$

Remark 1. Since $\emptyset \subset\{1, \ldots, n\}$, we can consider that $T_{\emptyset} f=f(0, \ldots, 0)$, that is, $T_{\emptyset} f$ can be considered as one of the functions in (2). If we accept this notation, then $\|f\|_{*}=\sum_{S \subseteq\{1, \ldots, n\}}\left\|T_{S} f\right\|_{\mathcal{L}_{\alpha}^{p}}$. 
Remark 2. To be more suggestive we explain here what condition (2) exactly means when $n=3$. In this case it means that the following eight functions are in $\mathcal{L}_{\alpha}^{p}\left(U^{3}\right)$ :

$$
\begin{array}{ll}
f(0,0,0), & \left(1-\left|z_{1}\right|^{2}\right)\left(1-\left|z_{2}\right|^{2}\right) \frac{\partial^{2} f\left(z_{1}, z_{2}, 0\right)}{\partial z_{1} \partial z_{2}} \\
\left(1-\left|z_{1}\right|^{2}\right) \frac{\partial f\left(z_{1}, 0,0\right)}{\partial z_{1}}, & \left(1-\left|z_{1}\right|^{2}\right)\left(1-\left|z_{3}\right|^{2}\right) \frac{\partial^{2} f\left(z_{1}, 0, z_{3}\right)}{\partial z_{1} \partial z_{3}} \\
\left(1-\left|z_{2}\right|^{2}\right) \frac{\partial f\left(0, z_{2}, 0\right)}{\partial z_{2}}, & \left(1-\left|z_{2}\right|^{2}\right)\left(1-\left|z_{3}\right|^{2}\right) \frac{\partial^{2} f\left(0, z_{2}, z_{3}\right)}{\partial z_{2} \partial z_{3}} \\
\left(1-\left|z_{3}\right|^{2}\right) \frac{\partial f\left(0,0, z_{3}\right)}{\partial z_{2}}, & \left(1-\left|z_{1}\right|^{2}\right)\left(1-\left|z_{2}\right|^{2}\right)\left(1-\left|z_{3}\right|^{2}\right) \frac{\partial^{3} f\left(z_{1}, z_{2}, z_{3}\right)}{\partial z_{1} \partial z_{2} \partial z_{3}} .
\end{array}
$$

\section{Proof of the main result}

To prove the main result we need an auxiliary result which is incorporated in the following theorem.

Theorem C. Suppose $0<p<\infty$ and $\alpha>-1$. Then there is a constant $C=C(p, \alpha)$ such that

$$
\int_{U}|f(z)|^{p}\left(1-|z|^{2}\right)^{\alpha} d m(z) \leq C\left(|f(0)|^{p}+\int_{U}\left|f^{\prime}(z)\right|^{p}\left(1-|z|^{2}\right)^{p+\alpha} d m(z)\right)
$$

for all $f \in H(U)$.

This theorem is a special case of Theorem 1 in [10]. Another proof of this theorem can be found in [14].

Proof of Theorem 1. Necessity. The proof of this part of the theorem is a special case of the proof of Theorem 1 in [15].

Sufficiency. First, assume that $n=2$. Let $z_{2}$ be, for a moment, fixed. Then by Theorem $\mathrm{C}$ we have

$$
\begin{aligned}
& \int_{U}\left|f\left(z_{1}, z_{2}\right)\right|^{p}\left(1-\left|z_{1}\right|^{2}\right)^{\alpha_{1}} d m\left(z_{1}\right) \\
& \leq C\left(\left|f\left(0, z_{2}\right)\right|^{p}+\int_{U}\left|\frac{\partial f}{\partial z_{1}}\left(z_{1}, z_{2}\right)\right|^{p}\left(1-\left|z_{1}\right|^{2}\right)^{p+\alpha_{1}} d m\left(z_{1}\right)\right)
\end{aligned}
$$

Multiplying this inequality by $\left(1-\left|z_{2}\right|^{2}\right)^{\alpha_{2}} d m\left(z_{2}\right)$, integrating over $U$ and ap- 
plying Fubini's theorem we obtain

$$
\begin{aligned}
\int_{U^{2}}\left|f\left(z_{1}, z_{2}\right)\right|^{p}\left(1-\left|z_{1}\right|^{2}\right)^{\alpha_{1}}\left(1-\left|z_{2}\right|^{2}\right)^{\alpha_{2}} d m\left(z_{1}\right) d m\left(z_{2}\right) \\
\leq C\left(\int_{U}\left|f\left(0, z_{2}\right)\right|^{p}\left(1-\left|z_{2}\right|^{2}\right)^{\alpha_{2}} d m\left(z_{2}\right)\right. \\
\left.\quad+\int_{U}\left(\int_{U}\left|\frac{\partial f}{\partial z_{1}}\left(z_{1}, z_{2}\right)\right|^{p}\left(1-\left|z_{2}\right|^{2}\right)^{\alpha_{2}} d m\left(z_{2}\right)\right)\left(1-\left|z_{1}\right|^{2}\right)^{p+\alpha_{1}} d m\left(z_{1}\right)\right) .
\end{aligned}
$$

On the other hand, for a fixed $z_{1}$ by Theorem $\mathrm{C}$ we have

$$
\begin{aligned}
& \int_{U}\left|\frac{\partial f}{\partial z_{1}}\left(z_{1}, z_{2}\right)\right|^{p}\left(1-\left|z_{2}\right|^{2}\right)^{\alpha_{2}} d m\left(z_{2}\right) \\
& \quad \leq C\left(\left|\frac{\partial f}{\partial z_{1}}\left(z_{1}, 0\right)\right|^{p}+\int_{U}\left|\frac{\partial^{2} f}{\partial z_{1} \partial z_{2}}\left(z_{1}, z_{2}\right)\right|^{p}\left(1-\left|z_{2}\right|^{2}\right)^{p+\alpha_{2}} d m\left(z_{2}\right)\right) .
\end{aligned}
$$

Let

$$
I=\int_{U^{2}}\left|f\left(z_{1}, z_{2}\right)\right|^{p}\left(1-\left|z_{1}\right|^{2}\right)^{\alpha_{1}}\left(1-\left|z_{2}\right|^{2}\right)^{\alpha_{2}} d m\left(z_{1}\right) d m\left(z_{2}\right) .
$$

From (3) and (4) and Fubini's theorem, we obtain

$$
\begin{aligned}
I \leq & C\left(\int_{U}\left|f\left(0, z_{2}\right)\right|^{p}\left(1-\left|z_{2}\right|^{2}\right)^{\alpha_{2}} d m\left(z_{2}\right)\right. \\
& +\int_{U}\left|\frac{\partial f}{\partial z_{1}}\left(z_{1}, 0\right)\right|^{p}\left(1-\left|z_{1}\right|^{2}\right)^{p+\alpha_{1}} d m\left(z_{1}\right) \\
& \left.\times \int_{U^{2}}\left|\frac{\partial^{2} f}{\partial z_{1} \partial z_{2}}\left(z_{1}, z_{2}\right)\right|^{p}\left(1-\left|z_{1}\right|^{2}\right)^{p+\alpha_{1}}\left(1-\left|z_{2}\right|^{2}\right)^{p+\alpha_{2}} d m\left(z_{1}\right) d m\left(z_{2}\right)\right) .
\end{aligned}
$$

Applying Theorem $\mathrm{C}$ to the function $f\left(0, z_{2}\right)$ we obtain

$$
\begin{aligned}
\int_{U}\left|f\left(0, z_{2}\right)\right|^{p} & \left(1-\left|z_{2}\right|^{2}\right)^{\alpha_{2}} d m\left(z_{2}\right) \\
& \leq C\left(|f(0,0)|^{p}+\int_{U}\left|\frac{\partial f}{\partial z_{2}}\left(0, z_{2}\right)\right|^{p}\left(1-\left|z_{2}\right|^{2}\right)^{p+\alpha_{2}} d m\left(z_{2}\right)\right) .
\end{aligned}
$$

From (5) and (6) the result follows in this case.

For $n \geq 3$ we use induction. Assume that we have proved the theorem for $k \leq n-1$, where $k$ is the number of variables. Let $z^{\prime}=\left(z_{1}, \ldots z_{n-1}\right)$. Then by 
inductive hypothesis we have

$$
\begin{aligned}
\int_{U^{n}}|f(z)|^{p} \prod_{j=1}^{n}\left(1-\left|z_{j}\right|^{2}\right)^{\alpha_{j}} d m\left(z_{j}\right) \\
=\int_{U}\left(\int_{U^{n-1}}\left|f\left(z^{\prime}, z_{n}\right)\right|^{p} \prod_{j=1}^{n-1}\left(1-\left|z_{j}\right|^{2}\right)^{\alpha_{j}} d m\left(z_{j}\right)\right)\left(1-\left|z_{n}\right|^{2}\right)^{\alpha_{n}} d m\left(z_{n}\right) \\
\leq \int_{U}\left(\left|f\left(0^{\prime}, z_{n}\right)\right|^{p}\right. \\
\quad+\sum_{S^{\prime} \subseteq\{1, \ldots, n-1\}} \int_{U^{\left|S^{\prime}\right|}}\left|\frac{\partial^{\left|S^{\prime}\right|} f}{\prod_{j \in S^{\prime}} \partial z_{j}}\left(\chi_{S^{\prime}}(1) z_{1}, \ldots, \chi_{S^{\prime}}(n-1) z_{n-1}, z_{n}\right)\right|^{p} \\
\left.\quad \times \prod_{j \in S^{\prime}}\left(1-\left|z_{j}\right|^{2}\right)^{\alpha_{j}+p} d m\left(z_{j}\right)\right)\left(1-\left|z_{n}\right|^{2}\right)^{\alpha_{n}} d m\left(z_{n}\right) .
\end{aligned}
$$

On the other hand we have

$$
\begin{aligned}
& \int_{U}\left|\frac{\partial^{\left|S^{\prime}\right|} f}{\prod_{j \in S^{\prime}} \partial z_{j}}\left(\chi_{S^{\prime}}(1) z_{1}, \ldots, \chi_{S^{\prime}}(n-1) z_{n-1}, z_{n}\right)\right|^{p} \prod_{j \in S^{\prime}}\left(1-\left|z_{n}\right|^{2}\right)^{\alpha_{n}} d m\left(z_{n}\right) \\
& \leq C\left(\left|\frac{\partial^{\left|S^{\prime}\right|} f}{\prod_{j \in S^{\prime}} \partial z_{j}}\left(\chi_{S^{\prime}}(1) z_{1}, \ldots, \chi_{S^{\prime}}(n-1) z_{n-1}, 0\right)\right|^{p}\right. \\
&+\int_{U}\left|\frac{\partial^{\left|S^{\prime}\right|+1} f}{\prod_{j \in S^{\prime}} \partial z_{j} \cdot \partial z_{n}}\left(\chi_{S^{\prime}}(1) z_{1}, \ldots, \chi_{S^{\prime}}(n-1) z_{n-1}, z_{n}\right)\right|^{p} \\
&\left.\times\left(1-\left|z_{n}\right|^{2}\right)^{\alpha_{n}+p} d m\left(z_{n}\right)\right) .
\end{aligned}
$$

Applying Fubini's theorem in (7), then substituting (8) in the obtained inequality, using the inequality

$\int_{U}\left|f\left(0^{\prime}, z_{n}\right)\right|^{p}\left(1-\left|z_{n}\right|^{2}\right)^{\alpha_{n}} \leq C\left(|f(\overrightarrow{0})|^{p}+\int_{U}\left|\frac{\partial f}{\partial z_{n}}\left(0^{\prime}, z_{n}\right)\right|^{p}\left(1-\left|z_{n}\right|^{2}\right)^{p+\alpha_{n}} d m\left(z_{n}\right)\right)$

and the fact that every $S \subseteq\{1, \ldots, n\}$ can be written as $S=S^{\prime} \cup\{\emptyset\}$ or $S=S^{\prime} \cup\{n\}$ where $S^{\prime}$ is a subset of $\{1, \ldots, n-1\}$, we obtain the result.

Remark 3. Throughout the proof we use the letter $C$ for a constant which may vary from line to line.

Corollary 1. Let $p \in(0, \infty)$ and $f \in H\left(U^{n}\right)$ be such that $f(z)=0$ whenever $\prod_{j=1}^{n} z_{j}=0$. Then, for some $C>0$ independent of $f$ it holds

$$
\int_{U^{n}}|f(z)|^{p} \prod_{j=1}^{n}\left(1-\left|z_{j}\right|^{2}\right)^{\alpha_{j}} d m\left(z_{j}\right) \leq C \int_{U^{n}}\left|\frac{\partial^{n} f}{\partial z_{1} \cdots \partial z_{n}}(z)\right| \prod_{j=1}^{p}\left(1-\left|z_{j}\right|^{2}\right)^{\alpha_{j}+p} d m\left(z_{j}\right) \text {. }
$$


By Theorem A and Theorem 1 we see that the following result is true.

Corollary 2. Let $p \in(0, \infty), \alpha=\left(\alpha_{1}, \ldots, \alpha_{n}\right)$, with $\alpha_{j}>-1$ for $j=1, \ldots, n$, let $m$ be a fixed positive integer and $\mathbf{k}=\left(k_{1}, \ldots, k_{n}\right) \in\left(\mathbf{Z}_{+}\right)^{n}$. Let $f \in H\left(U^{n}\right)$, then the following conditions are equivalent:

(a) $f \in \mathcal{A}_{\alpha}^{p}$

(b) $\left[\prod_{j=1}^{n}\left(1-\left|z_{j}\right|^{2}\right)^{k_{j}}\right] \frac{\partial^{|\mathbf{k}|} f}{\partial z_{1}^{k_{1}} \ldots \partial z_{n}^{k_{n}}}(z) \in \mathcal{L}_{\alpha}^{p} \quad \forall \mathbf{k},|\mathbf{k}|=m$;

(c) For every $S \subseteq\{1,2, \ldots, n\}$, the functions

$$
T_{S} f=\prod_{j \in S}\left(1-\left|z_{j}\right|^{2}\right) \frac{\partial^{|S|} f}{\prod_{j \in S} \partial z_{j}}\left(\chi_{S}(1) z_{1}, \chi_{S}(2) z_{2}, \ldots, \chi_{S}(n) z_{n}\right),
$$

are in $\mathcal{L}_{\alpha}^{p}\left(U^{n}\right)$.

Moreover,

$$
\begin{aligned}
\|f\|_{\mathcal{A}_{\alpha}^{p}} & \asymp \sum_{|\mathbf{k}|=0}^{m-1}\left|\frac{\partial^{|\mathbf{k}|} f}{\partial z_{1}^{k_{1}} \cdots \partial z_{n}^{k_{n}}}(0)\right|+\sum_{|\mathbf{k}|=m}\left\|\left[\prod_{j=1}^{n}\left(1-\left|z_{j}\right|^{2}\right)^{k_{j}}\right] \frac{\partial^{m} f}{\partial z_{1}^{k_{1}} \cdots \partial z_{n}^{k_{n}}}\right\| \|_{\mathcal{L}_{\alpha}^{p}} \\
& \asymp\|f\|_{*} .
\end{aligned}
$$

\section{References}

[1] G. Benke and D. C. Chang: A note on weighted Bergman spaces and the Cesáro operator. Nagoya Math. J. 159 (2000), 25 - 43.

[2] P. Duren: Theory of $H^{p}$ Spaces. New York: Academic Press 1970.

[3] T. M. Flet: The dual of an inequality of Hardy and Littlewood and some related inequalities. J. Math. Anal. Appl. 38 (1972), 746 - 765.

[4] G. H. Hardy and J. E. Littlewood: Some properties of fractional integrals II. Math. Z. 34 (1932), $403-439$.

[5] P. Lin and R. Rochberg: Henkel operators on the weighted Bergman spaces with exponential weights. Integral Equations Operator Theory 21 (1995), 460 $-483$.

[6] D. Luecking: Forward and reverse Carleson inequalities for functions in Bergman spaces and their derivatives. Amer. J. Math. 107 (1985), 85 - 111.

[7] M. Nowak: Bloch space on the unit ball of $\mathbf{C}^{n}$. Ann. Acad. Sci Fenn. Math. 23 (1998), $461-473$. 
[8] Caiheng Ouyang, Weisheng Yang and Ruhan Zhao: Characterizations of Bergman spaces and Bloch space in the unit ball of $\mathbf{C}^{n}$. Trans. Amer. Math. Soc. 347 (1995), $4301-4313$.

[9] W. Rudin, Function Theory in the Unit Ball of $C^{n}$. Berlin-Heidelberg-New York: Springer-Verlag 1980.

[10] Shi Ji-Huai: Inequalities for the integral means of holomorphic functions and their derivatives in the unit ball of $\mathbf{C}^{n}$. Trans. Amer. Math. Soc. 328 (1991) (2), $619-637$.

[11] A. Siskakis: Weighted integrals of analytic functions. Acta Sci. Math. 66 (2000), $651-664$.

[12] S. Stević: A note on weighted integrals of analytic functions. Bull. Greek Math. Soc. 46 (2002), 3 - 9.

[13] S. Stević: Weighted integrals of harmonic functions. Studia Sci. Math. Hung. 39 (2002)(1-2), $87-96$.

[14] S. Stević: The generalized Cesàro operator on Dirichlet spaces. Studia Sci. Math. Hung. 40 (2003) (1-2), 83 - 94.

[15] S. Stević: Weighted integrals of holomorphic functions on the polydisk. Z. Anal. Anwendungen 23 (2004) (3), 577 - 587.

[16] K. J. Wirths and J. Xiao: An image-area inequality for some planar holomorphic maps. Results Math. 38 (2000) (1-2), 172 - 179.

[17] Kehe Zhu: The Bergman spaces, the Bloch spaces, and Gleason's problem. Trans. Amer. Math. Soc. 309 (1988) (1), 253 - 268.

[18] Kehe Zhu: Duality and Hankel operators on the Bergman spaces of bounded symmetric domains. J. Funct. Anal. 81 (1988), 260 - 278.

[19] Kehe Zhu: Operator theory in function spaces. Pure and Applied Mathematics 136. New York-Basel: Marcel Dekker Inc. 1990.

Received 14.04.2004 